\title{
A patient with left-sided inferior vena cava who received oblique lumbar interbody fusion surgery: a case report
}

\author{
Chen Liu' ${ }^{1,2}$, Jian Zhai ${ }^{3}$, Quan Yuan ${ }^{3}, Y u$ Zhang ${ }^{1,2}$ and Hongguang $X u^{1,2^{*}}$
}

\begin{abstract}
Background: Oblique lateral interbody fusion surgery has become increasingly popular for lumbar degenerative diseases. The oblique corridor is between the psoas muscle and the retroperitoneal vessels, and its use could result in decreased tissue trauma, minimal blood loss, and short operation times. Patients who undergo oblique lateral interbody fusion surgery are always placed in the right lateral position to avoid damage to the inferior vena cava, which is typically a right-sided vessel. There is a substantial risk of vascular injury during the operation if there are anatomical variations in the vessels.
\end{abstract}

Case presentation: A 77-year-old man, of the Han nationality, with lumbar spinal stenosis underwent stand-alone oblique lateral interbody fusion surgery. Transverse magnetic resonance imaging of the lumbar spine indicated that his inferior vena cava was left-sided. A three-dimensional reconstructed image of abdominal computed tomography angiography showed that the inferior vena cava was located on the left side. Finally, the surgeon decided to change the position of our patient from a right lateral position to a left lateral position before the surgery.

Conclusions: To date, this is the first reported case where a patient underwent oblique lateral interbody fusion surgery in a left lateral decubitus position due to a left-sided inferior vena cava. This case demonstrates that carefully reading radiological results is important for operation planning and avoiding anatomical complications.

Keywords: Oblique lateral interbody fusion, Abdominal CTA, Inferior vena cava, Abdominal aorta, Left lateral decubitus position

\section{Background}

Oblique lateral interbody fusion (OLIF) was first introduced for degenerative disc disease with minimal surgical trauma in 2012 [1]. Compared to previous fusion techniques, such as posterior lumbar interbody fusion (PLIF), anterior lumbar interbody fusion (ALIF), and extreme lateral interbody fusion (XLIF)/direct lateral interbody fusion (DLIF), OLIF is a direct mini-open approach via the psoas major muscle and abdominal aorta; for this surgery, it was decided that almost all patients would be placed in the right lateral decubitus position because the inferior vena cava (IVC) is typically a

\footnotetext{
*Correspondence: xuhgyjsyy@163.com

'Spine Research Center of Wannan Medical College, Wuhu 241001, Anhui, China

${ }^{2}$ Department of Orthopaedics, Yijishan Hospital of Wannan Medical College, No. 2 Zheshan West Road, Wuhu 241001, Anhui, China

Full list of author information is available at the end of the article
}

right-sided vessel. However, we report the first case of an adult with a left-sided IVC who underwent OLIF surgery with the use of a right abdominal oblique incision.

\section{Case presentation}

A 77-year-old man of the Han nationality complained of numbness of his lower limbs for more than 5 years. In addition, his walking distance was less than 100 meters. A neurological examination suggested tenderness in the area of the lumbosacral spinous processes and bilateral paravertebral muscle. The feeling of numbness on the superficial skin of his bilateral lower limbs decreased. His Oswestry Disability Index (ODI) score was 24 and his Visual Analogue Scale (VAS) score was 5 before the operation. He had no history of hypertension, diabetes, or surgery. He also had no tobacco smoking or alcohol history. He took celecoxib orally, twice a day, one tablet a time; his pain was obviously not relieved. Before the 
operation, the white blood cell of our patient was $5.5 \times$ $10^{9} / \mathrm{L}$, the red blood cell was $4.26 \times 10^{12} / \mathrm{L}$, the hemoglobin (HGB) was $129 \mathrm{~g} / \mathrm{L}$, and the platelets (PLT) was $170 \times 10^{9} / \mathrm{L}$. As for his liver and renal functions, his alanine aminotransferase (ALT) was $11 \mathrm{U} / \mathrm{L}$ and his aspartate aminotransferase (AST) was $2211 \mathrm{U} / \mathrm{L}$. His urea was $6.07 \mathrm{mmol} / \mathrm{L}$ and his creatinine was $71.9 \mathrm{umol} / \mathrm{L}$. The tests of urine analysis, serology, and microbiology were normal.

Lumbar magnetic resonance imaging (MRI) (Fig. 1) indicated that the spinal canal of L4/5 was extremely narrow. He was diagnosed as having spondylolisthesis and lumbar spinal stenosis and was ready for stand-alone OLIF surgery in terms of clinical manifestations and imaging examinations. Unlike most other people, in this case, his IVC was discovered to be left-sided from the MRI image. In addition, we confirmed that he had a leftsided IVC with reconstruction computed tomography angiography (CTA) of the retroperitoneal vessels and lumbar vertebrae (Fig. 2). Finally, he underwent L4/5 stand-alone OLIF surgery under general anesthesia in the left lateral decubitus position. In the left lateral position, a transverse skin incision of $5 \mathrm{~cm}$ was made at the area of the right side of his abdomen, which was in the same horizontal plane as the L4/5 intervertebral disc (Fig. 3). His abdominal wall muscles were bluntly separated. The retroperitoneum was entered via blunt separation with the fingers; then, the psoas muscle was retracted posteriorly, and the abdominal vessels were retracted anteriorly. A guidewire was inserted in the middle of the target intervertebral disc with the help of a $\mathrm{C}$-arm. Sequential dilators were placed over the guidewire; then, a lighted retractor was placed over the dilators and fixed to the vertebral body with a pin, and the operation field was exposed. The annulus fibrosus and the nucleus pulposus were removed with a nucleus pulposus clamp. Then, the cartilage endplates were resected for exposure of the bony endplates. A wide and lordotic intervertebral fusion cage of $14 \times 55 \times 6 \mathrm{~mm}$ (Medtronic Clydesdale, Memphis, Tennessee) packed with allograft bone was inserted into the L4/5 disc with the guidance of the C-arm. Then, the incision was closed layer by layer. The blood loss was approximately $30 \mathrm{ml}$.

Our patient began to practice walking the day after the operation with waist protection, and his symptoms improved. A lumbar X-ray (Fig. 4a, b) after the surgery showed that the cage was located in the center, and he was discharged from our hospital 2 days after the surgery. After 3 months, the symptoms of lumbar and leg pain were significantly alleviated, and his ODI and VAS scores were 20 and 3, respectively. A lumbar X-ray also indicated that the location of the cage was perfect (Fig. $4 c, d)$. Half a year after the surgery, our patient did not complain of low back and leg pain. In addition, his ODI score was 19 and his VAS score was 2.

\section{Discussion}

At present, there are a number of studies on OLIF for the treatment of lumbar degenerative diseases, such as spondylolisthesis, lumbar spinal stenosis, and spinal deformity [2, 3]. Although OLIF reduces the risks of direct damage to the dural sac and the nerve root compared with traditional PLIF or transforaminal lumbar interbody fusion (TLIF), OLIF is associated with catastrophic complications, such as large retroperitoneal vessels, due to the limited operation approach between the psoas muscle and retroperitoneal vessels. It is very important for surgeons to have a good understanding of the anatomical relationship between the retroperitoneal vessels and the vertebral bodies to minimize the risks for these complications. The oblique access corridor has been investigated by many researchers. Liu et al. [4] selected imaging data from 60 adults who underwent abdominal CTA and T12-S1 vertebral computed tomography (CT) with three-dimensional reconstruction, and the vascular window, bare window, and psoas major window were
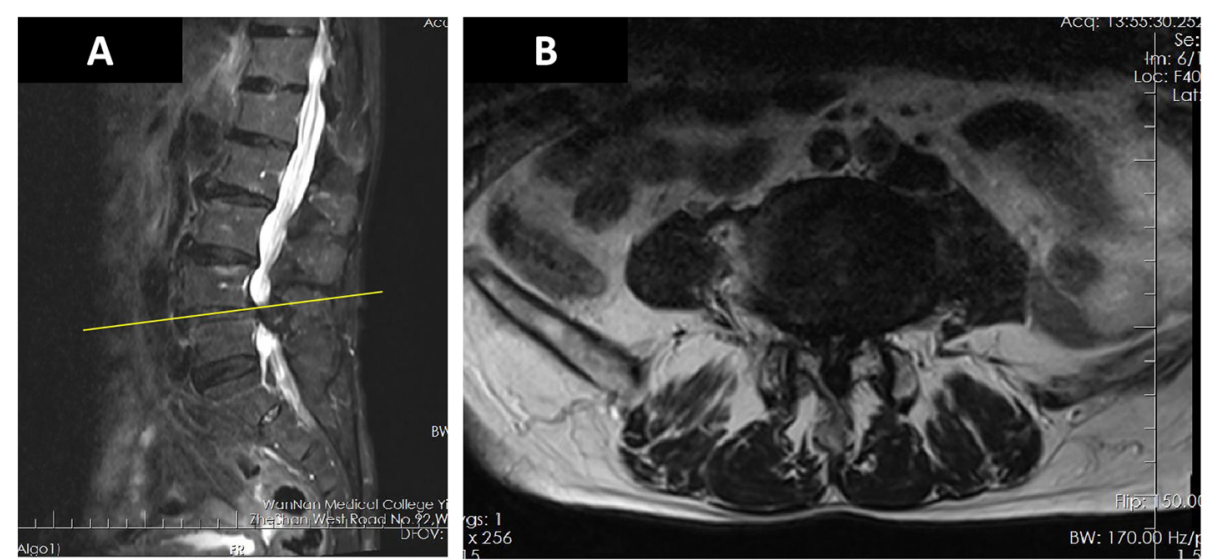

Fig. 1 Sagittal (a) and transverse (b) fat-suppressed magnetic resonance imaging of the lumbar spine indicated that the spinal canal was extremely narrow 


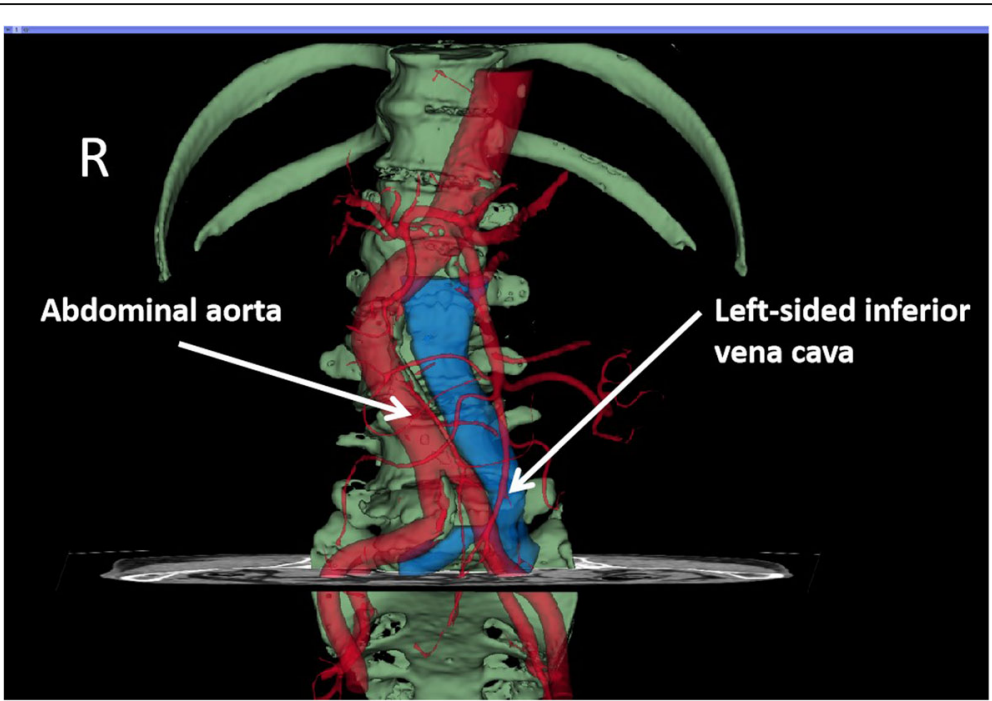

Fig. 2 A reconstruction computed tomography angiography of retroperitoneal vessels and lumbar vertebrae indicated that the inferior vena cava was located on the left side

measured. Davis et al. [5] dissected 20 fresh-frozen fulltorso cadaveric specimens and examined the oblique anatomical corridor of the L2-S1 discs. All measurements of the anatomical corridor were defined as the left lateral border of the aorta (or iliac artery). Almost all patients were placed in the right lateral position to receive the operation. This was the first report of using the left lateral position due to a left-sided IVC.

Variations in the location of the IVC are common. To date, there have been more than 60 kinds of congenital malformations of the IVC, including duplication, interruption, and transposition anomalies. Dual IVC is the most common variant of IVC anomaly. It results from the persistence of both supracardinal veins. The incidence of duplicated IVC accounts for $0.2-0.5 \%$ in the general population $[6,7]$. Other variations include a lack of a hepatic segment of the IVC from the azygos vein, a left renal vein from the circumferential aorta, complete absence of the IVC, and so on. Among the various kinds of anatomical variations, a left-sided IVC is extremely rare. Ang et al. [8] investigated published studies of left-sided IVC between 2000 and 2011 and found that the prevalence of a left-sided IVC ranged from $0.1 \%$ to $0.4 \%$.

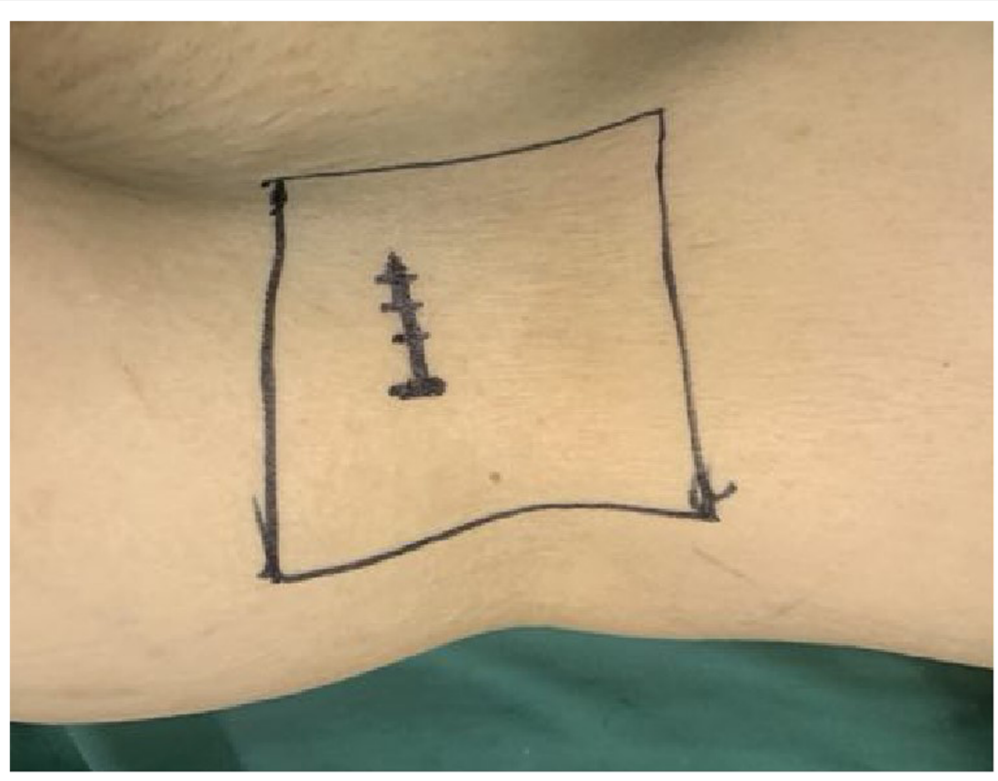

Fig. 3 A transverse incision was marked on the skin before the oblique lateral interbody fusion surgery 

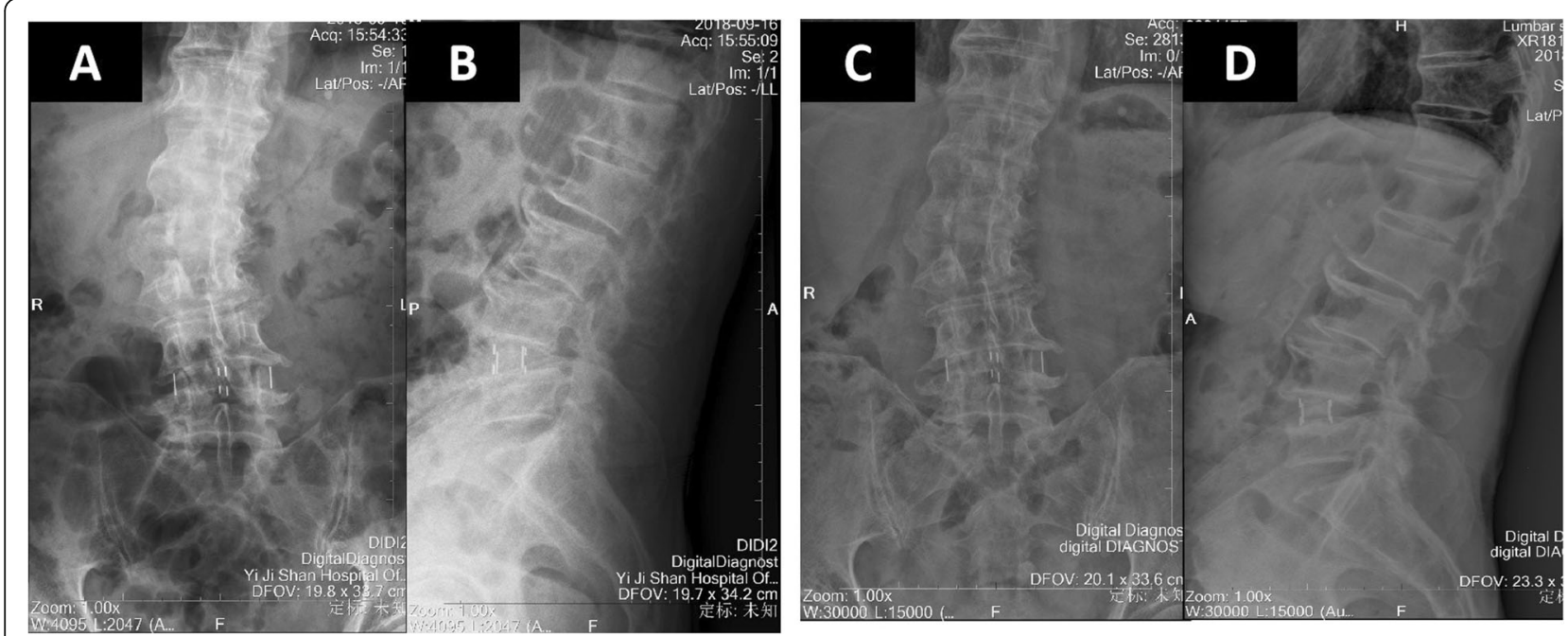

Fig. 4 X-ray of the lumbar spine after the stand-alone oblique lateral interbody fusion operation $(\mathbf{a}, \mathbf{b})$ and three-month follow-up (c, $\mathbf{d}$ )

The embryonic development of the IVC is complex. The IVC is formed by the successive development and degeneration of the posterior main vein, the inferior main vein, and the superior main vein. During this process, any abnormal development or normal degeneration can lead to different forms of IVC malformations. For the diagnosis of a left-sided IVC, digital subtraction angiography (DSA) can accurately display the whole lesion and measure the pressure of the right atrium and the IVC. DSA can provide a reliable basis for positioning, qualitative diagnosis, and guiding interventional therapy. The course of the IVC and its anatomical relationship with the abdominal aorta can also be well displayed by MRI and CT, especially with enhanced scans, to make a definitive diagnosis. For left-sided IVC malformations, there are no clinical symptoms or serious consequences. However, surgeons should pay attention to the operation of the related areas, and careful preoperative consideration should be taken to prevent intraoperative injuries.

In this case, the left-sided IVC was located between the abdominal aorta and the left psoas major muscle at the level of the L4/5 intervertebral disc, as shown in Fig. 5. Taking the possible vascular injury into consideration, we successfully completed the OLIF surgery through a right operative window to avoid damaging the variant IVC.

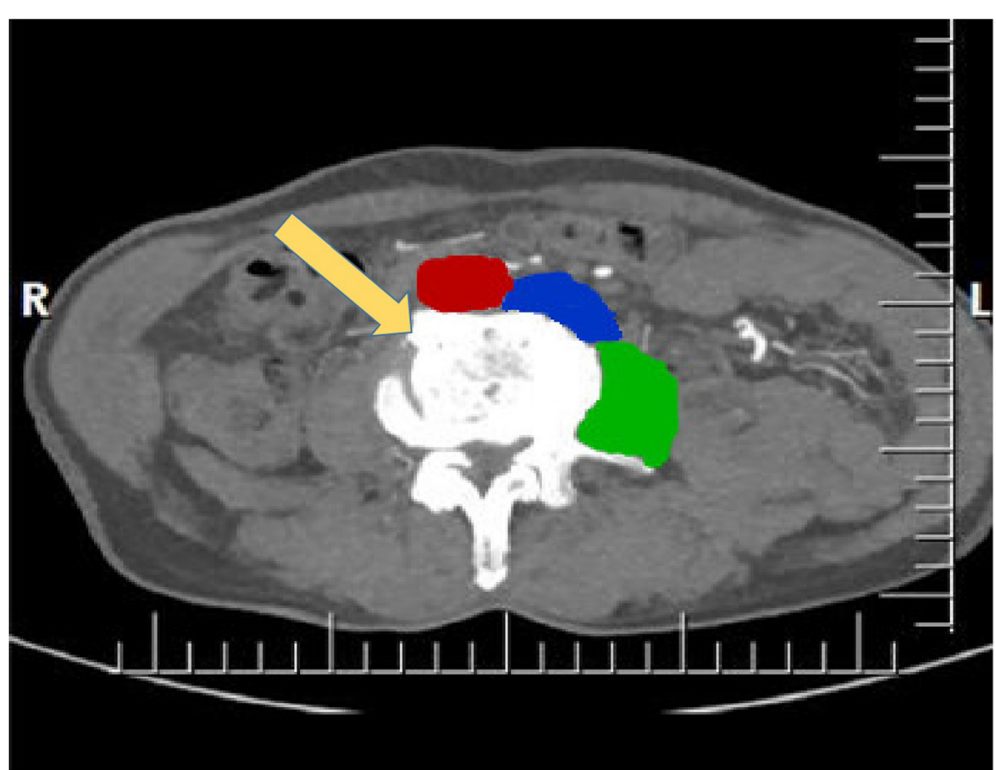

Fig. 5 A transverse image of a contrast-enhanced abdominal computed tomography scan. The red region refers to the abdominal aorta; the blue region represents the inferior vena cava; and the green region represents the psoas major muscle. The yellow arrow represents the operation window 


\section{Conclusions}

In fact, a left-sided IVC is uncommon but clinically significant. Up to now, this is the first reported case of a patient who was diagnosed as having lumbar spinal stenosis and underwent stand-alone OLIF surgery in the left lateral decubitus position due to the presence of a leftsided IVC. Although we did not consult the vascular service in this case, it is recommended that they should be involved when there are major vascular anomalies present. OLIF can be possible from both sides by an expert surgeon as there are no major technical challenges; however, vascular anomalies and variations must be kept in mind and radiologically identified. Awareness of variations in the IVC is crucial for the choice of an operation window during OLIF surgery, which could avoid many anatomically relevant complications. This patient may offer a good example of anatomical variations; surgeons should be aware of anatomical variations in vessels before performing operations.

\section{Abbreviations}

ALIF: Anterior lumbar interbody fusion; ALT: Alanine aminotransferase; AST: Aspartate aminotransferase; CT: Computed tomography; CTA: Computed tomography angiography; DLIF: Direct lateral interbody fusion; DSA: Digital subtraction angiography; HGB: Hemoglobin; IVC: Inferior vena cava; MRI: Magnetic resonance imaging; ODI: Oswestry Disability Index; OLIF: Oblique lateral interbody fusion; PLIF: Posterior lumbar interbody fusion; PLT: Platelets; TLIF: Transforaminal lumbar interbody fusion; VAS: Visual Analogue Scale; XLIF: Extreme lateral interbody fusion

\section{Acknowledgements}

Thanks to the National Nature Science Foundation of China.

\section{Authors' contributions}

$\mathrm{CL}$ is a doctor in the department of orthopaedics, Yijishan Hospital of Wannan Medical College, and he made substantial contributions to this study. JZ and QY are doctors in the department of radiology, Yijishan Hospital of Wannan Medical College, and they analyzed the CTA images. YZ participated in the critical discussion of the contents and drafting of the manuscript for publication. HX gave final approval after evaluating the contents and discussion. All authors read and approved the final manuscript.

\section{Funding}

The study was supported by the National Nature Science Foundation of China (81702158) and the National Nature Science Foundation of China (81572185).

\section{Availability of data and materials}

Not applicable.

\section{Ethics approval and consent to participate}

This study was approved by the Ethical Review Committee of Yijishan Hospital of Wannan Medical College (Wuhu, Anhui, China). The patient approved participation in this study.

\section{Consent for publication}

Written informed consent was obtained from the patient for publication of this case report and any accompanying images. A copy of the written consent is available for review by the Editor-in-Chief of this journal.

\section{Competing interests}

The authors declare that they have no competing interests.

\section{Author details}

${ }^{1}$ Spine Research Center of Wannan Medical College, Wuhu 241001, Anhui, China. ${ }^{2}$ Department of Orthopaedics, Yijishan Hospital of Wannan Medical College, No. 2 Zheshan West Road, Wuhu 241001, Anhui, China.

${ }^{3}$ Department of Radiology, Yijishan Hospital of Wannan Medical College, No. 2 Zheshan West Road, Wuhu 241001, Anhui, China.

Received: 4 April 2019 Accepted: 2 January 2020

Published online: 27 January 2020

References

1. Silvestre C, Mac-Thiong JM, Hilmi R, Roussouly P. Complications and Morbidities of Mini-open Anterior Retroperitoneal Lumbar Interbody Fusion: Oblique Lumbar Interbody Fusion in 179 Patients. Asian Spine J. 2012;6(2):89-97.

2. Abe K, Orita S, Mannoji C, Motegi H, Aramomi M, Ishikawa T, Kotani T, Akazawa T, Morinaga T. Perioperative Complications In 155 Patients Who Underwent Oblique Lateral Interbody Fusion Surgery: Perspectives and Indications From a Retrospective, Multicenter Survey. Spine. 2016;42(1):55-62.

3. Miscusi M, Ramieri A, Forcato $S$, Giuffrè M, Trungu S, Cimatti M. Comparison of pure lateral and oblique lateral interbody fusion for treatment of lumbar degenerative disk disease: a multicentric cohort study. Eur Spine J. 2018;10:1-7.

4. Liu L, Liang Y, Zhang H, Wang H, Guo C, Pu X, Zhang C, Wang L, Wang J, LV $Y$, Ren Z, Zhou Q, Deng Z. Imaging Anatomical Research on the Operative Windows of Oblique Lumbar Interbody Fusion. PLoS One. 2016;11(9): e0163452.

5. Davis T, Hynes RA, Fung DA, Spann SW, MacMillan M, Kwon B, Liu J, Acosta F, Drochner TE. Retroperitoneal oblique corridor to the L2-S1 intervertebral discs in the lateral position: an anatomic study. J Neurosurg Spine. 2014;21(5):785-93.

6. Cizginer S, Girshman J, Beckman JA, Silverman SG. Thrombosed interrupted inferior vena cava and retroaortic left renal vein mimicking retroperitoneal neoplasm. Abdom Imaging. 2007;32(3):403.

7. Gandhi SP, Modi P, Sutariya H, Patel K. Rare Anatomical Variation of Dual IVC with Left Sided IVC Draining into Hemiazygous Vein- A Case Report. J Clin Diagn Res. 2016;10(3):TD14

8. Ang WC, Doyle T, Stringer MD. Left-sided and duplicate inferior vena cava: A case series and review. Clin Anat. 2014;26(8):990-1001.

\section{Publisher's Note}

Springer Nature remains neutral with regard to jurisdictional claims in published maps and institutional affiliations.
Ready to submit your research? Choose BMC and benefit from:
- fast, convenient online submission
- thorough peer review by experienced researchers in your field
- rapid publication on acceptance
- support for research data, including large and complex data types
- gold Open Access which fosters wider collaboration and increased citations
- maximum visibility for your research: over $100 \mathrm{M}$ website views per year
At $\mathrm{BMC}$, research is always in progress.
Learn more biomedcentral.com/submissions 